\title{
Advanced Glycation End-Product Pentosidine Accumulates in Various Tissues of Rats with High Fructose Intake
}

\author{
K. MIKULÍKOVÁ ${ }^{1}$, A. ECKHARDT ${ }^{1,2}$, J. KUNES̆ ${ }^{1,2}$, J. ZICHA ${ }^{1,2}$, I. MIKŠÍK ${ }^{1,2}$ \\ ${ }^{1}$ Institute of Physiology, Academy of Sciences of the Czech Republic, Prague, ${ }^{2}$ Cardiovascular \\ Research Center, Prague, Czech Republic
}

Received August 17, 2006

Accepted January 18, 2007

On-line available February 8, 2007

\begin{abstract}
Summary
The slowly metabolized proteins of the extracellular matrix, typically collagen and elastin, accumulate reactive metabolites through uncontrolled non-enzymatic reactions such as glycation or the products arising from the reaction of unsaturated long chain fatty acid metabolites (possessing aldehydic groups). A typical example of these non-enzymatic changes is the formation of advanced glycation end-products (AGEs), resulting from the reaction of carbohydrates with the free amino group of proteins. The accumulation of AGEs and the resulting structural alterations cause altered tissue properties (increased stiffness, reduced elasticity) that contribute to their reduced catabolism and to their aging. Posttranslational nonenzymatic modifications of the proteins of the extracellular matrix (the formation of a typical AGE product - pentosidine) were studied in three types of tissue of three rat strains subjected to a high-fructose diet. Chronic (three-week) hyperglycemia (resulting from fructose loading) caused a significant increase in pentosidine concentration mainly in the aorta and skin of the three rat strains (Lewis, Wistar and hereditary hypertriglyceridemic rats).
\end{abstract}

\section{Key words}

Collagen • Pentosidine $\bullet$ Ages $\bullet$ Elastin $\bullet$ Glycation

\section{Corresponding author}

Adam Eckhardt, Institute of Physiology, Czech Academy of Sciences, Vídeňská 1083, Prague 4, 14220, Czech Republic. E-mail: eckhardt@biomed.cas.cz

\section{Introduction}

Proteins undergo important non-enzymatic (chemical) posttranslational modifications with a significant physiological/biological impact. The most susceptible proteins are the slowly metabolized ones, having a longer exposure time for chemical reactions with endo- and exogenous compounds (Maillard 1912, Trivelli et al. 1971).

The extracellular matrix is formed by these slowly metabolized proteins, typically collagen and elastin. One of the typical non-enzymatic reactions is glycation, the reaction between the reactive oxo-group of the sugar and amino group of the protein (Deyl and Mikšík 1997, Mikšík and Deyl 1997, Monnier 1989, Monnier et al. 1992). In this sequence of reactions, a number of compounds arise that are not yet completely known, including monotopically as well as multitopically (cross-link) bound products. This results in the formation of advanced glycation end-products (AGEs). The accumulation of AGEs and the resulting structural alterations alter tissue properties (higher stiffness, lower elasticity) that contribute to their reduced catabolism and to their aging. Collagen, which is a typical long-life protein, loses flexibility and enzymatic digestivity in parallel with aging (Schnider and Kohn 1981). Similar changes in collagen are known to occur in diabetic patients (Schnider and Kohn 1982). AGEs in mammals have been predominantly chemically characterized by the detection of fluorescent products - namely pentosidine (imidazo [4,5-b] pyridinium molecule comprising a lysine and arginine), one of a few presently known glycation products (Sell and Monnier 1989).

One of the most prevalent metabolic syndromes, diabetes mellitus, is a major risk factor for cardiovascular disease. Atherosclerosis accounts for most of the morbidity and mortality of diabetic patients. Diabetes 
mellitus is characterized by hyperglycemia, resulting in metabolic changes in lipid and protein metabolism and irreversible long-term vascular and connective tissue changes. These changes include diabetes-specific complications such as retinopathy, nephropathy and neuropathy and complications of the macrovasculature such as atherosclerosis, potentially resulting in heart disease, stroke and peripheral vascular disease (Hudson at al. 2001). AGEs in hyperglycemic patients are reviewed by Hatfield and Mulfinger (2005). Pentosidine has been found to accumulate in the aorta, tail and skin collagen matrix at accelerated rates in diabetic mammals (Kern and Engerman 2001, McCance et al. 1993, Nyengaard et al. 1997). Links between chronic hyperglycemia and the development of long-term diabetic-specific complications have been discovered but not yet completely understood (Hudson at al. 2001, Reddy 2004, Wautier and Guillausseau 2001).

The passive biomechanical properties of vessels change with age. An increase in thickness and stiffness, as well as a reduction in elasticity of the aorta during aging have been shown in both rats and humans (Bruel at al. 1998, Bruel and Oxlund 1996, Sonesson at al. 1997). These changes are primarily caused by smooth muscle cell hypertrophy and fibrosis. During aging and senescence, the concentration of collagen and elastin changes in the rat aorta (Bruel and Oxlund 1996). The elastin concentration decreases and the concentration of collagen (mainly collagen type III) increases up to a certain age and then reaches a plateau (Cox 1983, Cox and Detweiler 1988, Vogel 1991).

Cardiovascular diseases are in many cases accompanied by lipid disorders (Marin-Garcia and Goldenthal 2002). Arterial hypertension is a widely studied problem with a direct relationship to modifications of the vessel wall. Hypertension often results in an increased thickness and stiffness of large artery walls (Clerc et al. 1999, Karam et al. 1996, Laurent 1995, Laurent et al. 1994). The AGEs of plasma and matrix proteins are candidate mediators for various vascular complications such as atherosclerosis. A significantly larger accumulation of AGEs was detected in the aorta of stroke-prone spontaneously hypertensive rats (Mizutani at al. 1999).

In this study, hereditary hypertriglyceridemic (HTG) rats were used, which represent a genetic model of hypertension causing metabolic disturbances in lipid and carbohydrate metabolism (Štolba et al. 1992). This animal model seems to be suitable for studying the posttranslational products arising from both reactions with reducing sugars and with lipid-derived compounds. HTG rats were used because we assumed a possible influence of aldehydic intermediates of lipids on the formation of the AGEs namely pentosidine The pentosidine concentration in the skin and tail tendon collagen of HTG rats significantly increases with age (Mikšík at al. 1997).

The posttranslational non-enzymatic modifications of extracellular matrix proteins (the formation of the typical AGE - pentosidine) were studied in three types of tissues (skin, tail tendons and aortas) of three strains of rats (Wistar, Lewis and HTG) that were also subjected to a high fructose intake.

\section{Methods}

\section{Chemicals}

All chemicals used were of either analytical grade or the highest available purity. Trifluoroacetic acid and acetonitrile (HPLC gradient grade) were purchased from Merck (Darmstadt, Germany). All other chemicals were obtained from Sigma (St. Louis, MO, USA). All solutions were prepared in Milli-Q Water (Millipore, Bedford, MA, USA).

\section{Instrumental}

The HPLC apparatus used was a Agilent 1100 (Agilent, Fullerton, CA, USA) consisting of a degasser, a binary pump, a thermostated column compartment, diode array and fluorescence detectors, the column used was an Atlantis ( 150 x 3.9 mm I.D., $3 \mu \mathrm{m}, \mathrm{C}_{18}$, Waters, Ireland).

\section{Experimental animals}

Three strains of rats were used: a) six-month-old male HTG rats (originally selected from the Wistar rats as described by Vrána and Kazdová 1990), b) six-month-old male Wistar rats, and c) six-month-old male Lewis rats. All three strains of rats were split to two groups: 1) control rats (C) - were fed a standard rat chow, and 2) rats kept on high fructose intake $(10 \% \mathrm{~F})$ which drank $10 \%$ fructose solution for the last three weeks before being sacrified. Every group contained at least five animals.

\section{Sample preparation}

Three types of tissue samples (skin, tail tendons and aorta) were taken from all groups of rats. All samples were prepared according to a previously published 
procedure (Dunn at al. 1991). Briefly, after thawing, the skin samples were scraped vigorously with a scalpel to remove the adherent fat and other tissues (tendon and aorta samples were prepared without this step). All samples were extracted sequentially for $72 \mathrm{~h}$ at room temperature with chloroform-methanol (2:1), and $1 \mathrm{~mol} / \mathrm{l}$ $\mathrm{NaCl}$, to remove the lipids and soluble proteins. After the final extraction, the samples were rinsed in distilled water and dried by lyophilization.

\section{Preparation of the pentosidine standard}

The pentosidine standard was prepared by a modified method of Dyer et al. (1991). The pentosidine was purified in two subsequent separation steps by HPLC in a Supelcosil LC-318 column $(250$ x $4.6 \mathrm{~mm}$ i.d., $5 \mu \mathrm{m}$, $300 \AA$ pores). In the first step, separation was achieved by gradient elution using a water-acetonitrile gradient (with $0.1 \%$ TFA). In the second step, TFA was replaced by $0.1 \%$ HFBA. The purified product was deacetylated by heating in $6 \mathrm{M} \mathrm{HCl}$ for $1 \mathrm{~h}$ at $110^{\circ} \mathrm{C}$, the pentosidine was finally purified using a system with $0.1 \%$ HFBA. The identification of pentosidine was confirmed by measuring its fluorescence spectra.

\section{Amino acid analysis}

The method uses precolumn derivatization with phenylisothiocyanate (the PICO-TAG system, Waters, following the manufacturer's instructions) followed by the separation of the resulting products by HPLC in a reversed-phase column $\left(\mathrm{C}_{18}\right.$, Pico-Tag column $300 \times 3.9$ i.d. mm, Waters) using an acetate buffer ( $\mathrm{pH} 6.4$, $0.14 \mathrm{~mol} / \mathrm{l}$ )-acetonitrile gradient (following the manufacturer's procedure).

\section{Collagen and elastin assessment}

Collagen and elastin levels were assessed from the amino acid contents of glycine (about $33 \%$ of the residues in collagen and elastin), hydroxyproline (specific to collagen) and histidine (about $13 \%$ in elastin).

\section{Pentosidine measurement}

The hydrolysis of protein samples (skin, tail tendons and aorta) was carried out with $\mathrm{HCl}$ vapor ( $6 \mathrm{~mol} / \mathrm{l} \mathrm{HCl}$ with $2 \%$ phenol) for $20 \mathrm{~h}$ at $106^{\circ} \mathrm{C}$ in a vial under vacuum. After lyophilization of $200 \mu \mathrm{l}$ of hydrolysate, the sample was dissolved in $25 \mu \mathrm{l}$ of $2 \%$ heptafluorobutyric acid (HFBA) and $10 \mu \mathrm{l}$ was injected into the HPLC column (Atlantis: reversed-phase C18, $50 \times 3.9$ i.d. $\mathrm{mm}$, Waters). Elution was done using a

\section{Controls of Wistar, Lewis and HTG strain}

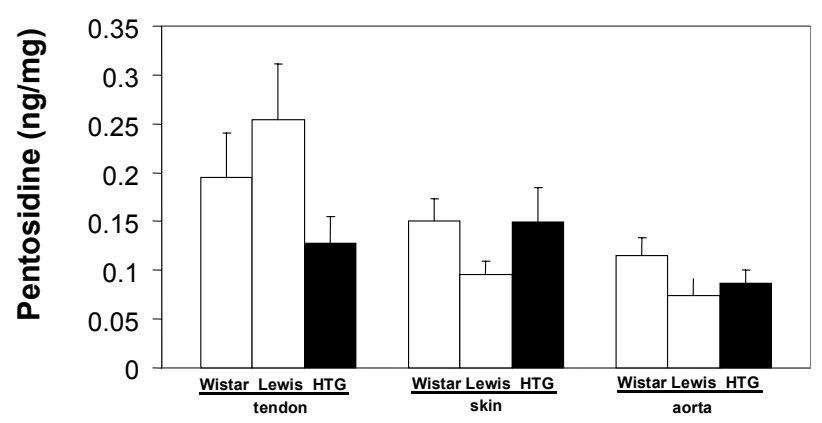

Fig. 1. Pentosidine content in control groups of rats: tail tendon, skin and aorta of HTG, Wistar, Lewis and HTG strains. Concentration is expressed as $\mathrm{ng} / \mathrm{mg}$ of total protein.

gradient of water with $0.1 \% \operatorname{HFBA}(\mathrm{A})$ and acetonitrile with $0.085 \%$ HFBA (B) from $0 \%$ B to $9 \%$ B at 2 min, gradient continued from $9 \%$ to $17 \%$ at $21 \mathrm{~min}$, and then from $17 \%$ to $100 \% \mathrm{~B}$ at $25 \mathrm{~min}$. After that, the column was equilibrated with $100 \% \mathrm{~A}$ for $10 \mathrm{~min}$. The flow rate was $0.8 \mathrm{ml} / \mathrm{min}$ and the column temperature was held at $40{ }^{\circ} \mathrm{C}$. The eluate was monitored by its fluorescence at $332_{\mathrm{ex}} / 378_{\mathrm{em}} \mathrm{nm}$. The concentration of pentosidine was expressed as nanograms per milligram of total protein.

\section{Evaluation}

All data are expressed as mean \pm SD. Statistical analyses of the data were performed and the probability values (P) for significance were calculated using Student's $t$-test. $\mathrm{P} \leq 0.05$ values were considered to be statistically significant.

\section{Results}

The amino acid analysis of samples did not reveal any significant differences in the content of collagen and elastin between the controls and fructosetreated animals in the examined tissues of any of the rat strains (data not shown). Elastin content was higher (about $30 \%$ of dry weight) in all aorta samples in comparison with skin and tail tendon tissues (in all rat strains) as expected, but there was no significant difference between the particular rat strains.

In these experiments we used Wistar, Lewis and HTG rats that represent a genetic model of hypertension with accompanying metabolic disturbances of lipid and carbohydrate metabolism (Štolba et al., 1992). It was assumed that these animal models are suitable for studying the posttranslational products arising from both the reaction with the oxo-groups of reducing sugars and 


\section{Lewis strain}

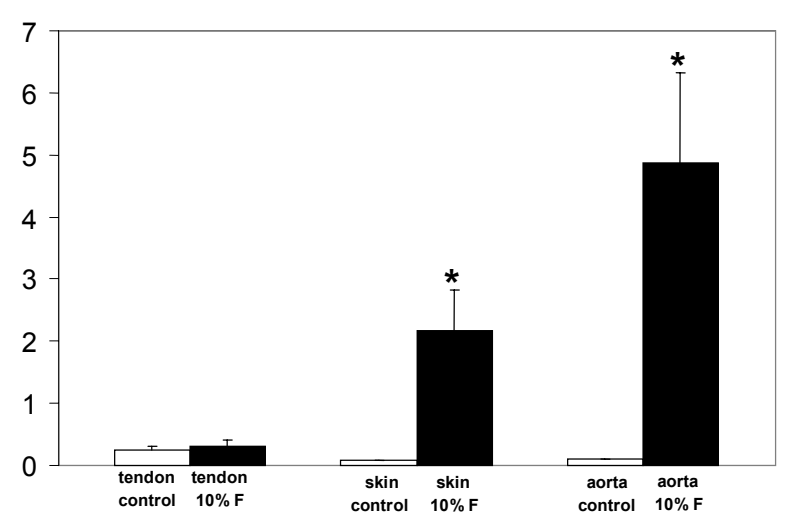

Fig. 2. Pentosidine content of tissues of Lewis rats: controls and rats kept on water containing $10 \%$ fructose solution (10\% F). Statistical significance: $* P<0.02$. Concentration is expressed as $\mathrm{ng} / \mathrm{mg}$ of total protein.

with lipid-derived compounds.

In water drinking rats, the tissue concentration of pentosidine did not significantly differ among the three strains of rats (Wistar, Lewis, HTG) (Fig. 1). Under these conditions, the concentration of blood glucose was also not significantly different in these rat strains (Kadlecová et al. 2004). From this point of view, it was not surprising that the level of pentosidine, the compound arising from the reaction of sugar and amino acid, was not significantly different.

The situation was different when the rats were drinking $10 \%$ fructose solution. The amounts of the posttranslational non-enzymatic product pentosidine significantly increased with high fructose intake in all three strains of rats in the aorta and skin samples, and in the tendon sample of the HTG strain (Figs. 2, 3 and 4). It should be noted that blood glucose levels were elevated by $60-80 \%$ in fructose-drinking animals compared to the water-drinking controls (Kadlecová et al. 2004). The amounts of pentosidine in the tail tendons of fructose drinking Wistar and Lewis rats were higher than in the respective controls, but the variability of the concentrations was too high.

\section{Discussion}

This paper is focused on pentosidine which is the only one known advanced glycation end-product crosslinking proteins. It could therefore have a strong influence on stiffness of the tissue (especially in blood vessels) and it could be an additional risk factor for their normal function.

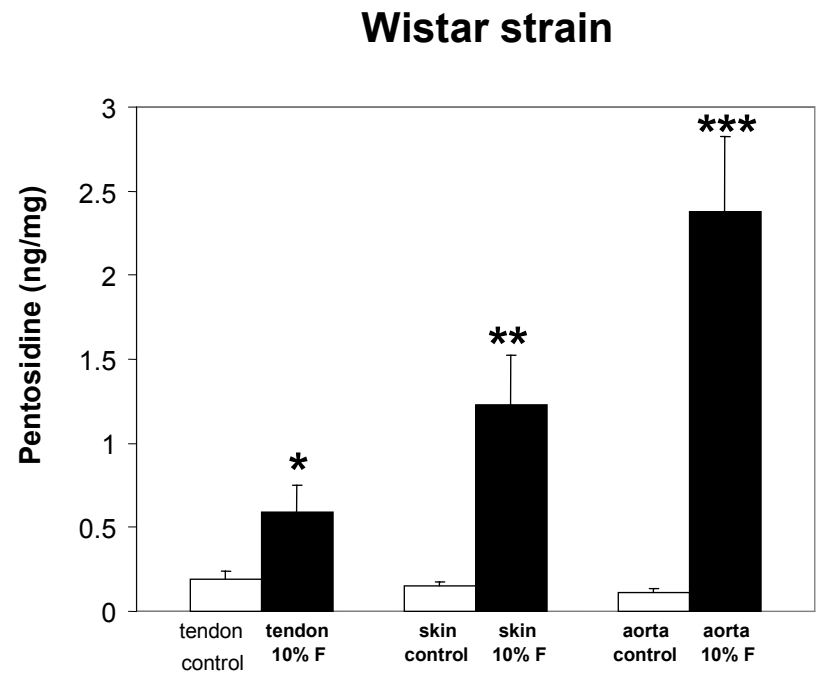

Fig. 3 Pentosidine content of tissues of Wistar rats: controls and rats kept on water containing $10 \%$ fructose solution (10\% F). Statistical significance: $* P<0.05$, $* * P<0.01$, and $* * * P<0.001$. Concentration is expressed as $\mathrm{ng} / \mathrm{mg}$ of total protein.

The amounts of pentosidine significantly increased with high fructose intake in the aorta and skin samples of all three strains of rats, although the pentosidine levels in tail tendons, aortas and skin of fructose-treated rats were highly variable. Our findings are in agreement with published results (Kern and Engerman 2001, McCance et al. 1993, Nyengaard et al. 1997), which indicated that pentosidine has been accumulated in the aortas of diabetic rats (Nyengaard et al. 1997) and dogs (Kern and Engerman 2001), in the skin of diabetic patients (McCance et al. 1993), and in the tail collagen of diabetic dogs (Kern and Engerman 2001).

The higher triglyceride content in the blood of HTG rats had no influence on the content of pentosidine in all three kinds of tendons. The changes in pentosidine concentrations were not significant in a comparison of HTG with Wistar, or HTG with Lewis rats. From this finding we can conclude that the level of pentosidine is not dependent on the level of triglycerides and/or their metabolic products.

There were no significant differences in the content of collagen and elastin between the controls and fructose-treated animals in any tissues of the examined rat strains. We hypothesize that the high fructose intake had no influence on the ratio of these proteins. It is possible that ratio between collagen types (I and III) can change. This determination of the collagen types is out of scope of this paper, but our group is currently developing a new method (based on HPLC-MS measurement). 


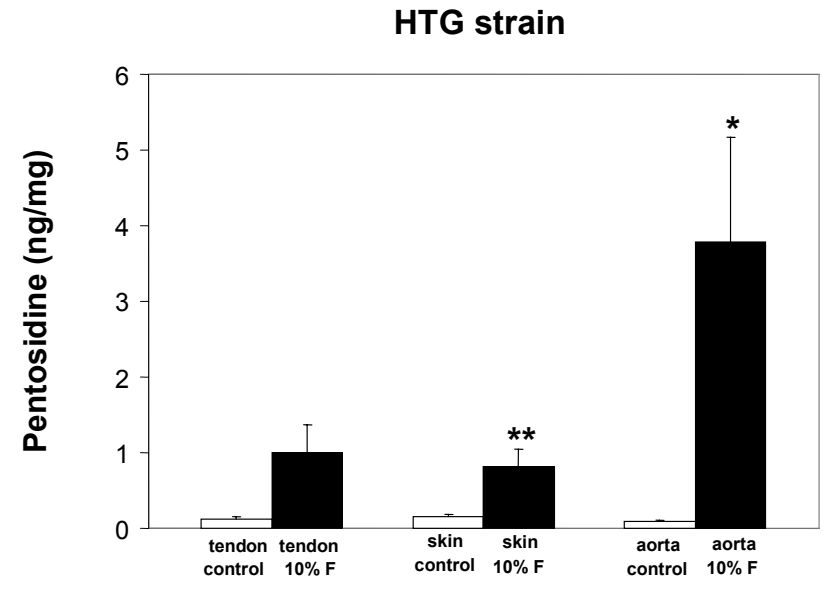

Fig. 4 Pentosidine content of tissues of HTG rats: controls and rats kept on water containing $10 \%$ fructose solution $(10 \% \mathrm{~F})$. Statistical significance: $* P<0.05, * * \quad P<0.02$. Concentration is expressed as $\mathrm{ng} / \mathrm{mg}$ of total protein.

HTG rats were used because we assumed a possible influence of aldehydic intermediates of lipids on the formation of the AGEs namely pentosidine. However, the skin, tail tendons and aortas of six month-old HTG rats had an unchanged extracellular matrix (as far as collagen and elastin content were concerned) and unchanged pentosidine content in comparison with Lewis and Wistar strain rats. No significant changes in the content of collagen and elastin were observed between the controls and rats kept on a high fructose intake. The higher triglyceride content in the blood of HTG rats had no influence on the content of pentosidine in all three kinds of tendons. Chronic (three-week) hyperglycemia caused a significant increase in the pentosidine concentration in the aorta and skin of three rat strains (Lewis, Wistar and HTG) and in the tail tendons of the Wistar strain. A higher pentosidine content was also observed in the tail tendons of Lewis and HTG rats, but these changes were not significant.

\section{Conflict of Interest}

There is no conflict of interest.

\section{Acknowledgements}

This work was supported by the Grant Agency of the Czech Republic, grants No. 203/05/2539, 305/08/0108, and the Centre for Cardiovascular Research 1M0510.

\section{References}

BRUEL A, OXLUND H: Changes in biomechanical properties, composition of collagen and elastin, and advanced glycation endproducts of the rat aorta in relation to age. Atherosclerosis 127: 155-165, 1996.

BRUEL A, ORTOFT G, OXLUND H: Inhibition of cross-links in collagen is associated with reduced stiffness of the aorta in young rats. Atherosclerosis 140: 135-145, 1998.

CLERC PC, RENAUD JF, BLACHER J, LEGRAND M, SAMUEL JL, LEVY BI: Collagen I and III and mechanical properties of conduit arteries in rats with genetic hypertension. J Vasc Res 36: 139-146, 1999.

COX RH: Age-related changes in arterial wall mechanics and composition of NIA Fischer rats. Mech Ageing Dev 23: 21-36, 1983.

COX RH, DETWEILER DK: Comparison of arterial wall properties in young and old racing greyhounds. Mech Ageing Dev 44: 51-67, 1988.

DEYL Z, MIKŠÍK I: Post-translational non-enzymatic modification of proteins.1. Chromatography of marker adducts with special emphasis to glycation reactions. J Chromatogr B 699: 287-309, 1997.

DUNN JA, MCCANCE DR, THORPE SR., LYONS TJ, BAYNES JW: Age-dependent accumulation of N epsilon(carboxymethyl)lysine and $\mathrm{N}$ epsilon-(carboxymethyl)hydroxylysine in human skin collagen. Biochemistry 30: 1205-1210, 1991.

DYER DG, BLACKLEDGE JA, THORPE SR, BAYNES JW: Formation of pentosidine during nonenzymatic browning of proteins by glucose. Identification of glucose and other carbohydrates as possible precursors of pentosidine in vivo. $J$ Biol Chem 266: 11654-11660, 1991.

HATFIELD J, MULFINGER L: Advanced glycation end-products (AGEs) in hyperglycemic patients. $J$ Young Investig 13: $\mathrm{id}=575,2005$.

HUDSON BI, STICKLAND MH, FUTERS TS, GRANT PJ: Effects of novel polymorphisms in the RAGE gene on transcriptional regulation and their association with diabetic retinopathy. Diabetes 50: 1505-1511, 2001.

KADLECOVÁ M, ČEJKA J, ZICHA J, KUNEŠ J: Does Cd36 gene play a key role in disturbed glucose and fatty acid metabolism in Prague hypertensive hypertriglyceridemic rats? Physiol Res 53: 265-271, 2004. 
KARAM H, HEUDES D, GONZALES MF, LOFFLER BM, CLOZEL M, CLOZEL JP: Respective role of humoral factors and blood pressure in aortic remodeling of DOCA hypertensive rats. Am J Hypertens 9: 991-998, 1996.

KERN TS, ENGERMAN, RL: Pharmacological inhibition of diabetic retinopathy: aminoguanidine and aspirin. Diabetes 50: 1636-1642, 2001.

LAURENT S: Arterial wall hypertrophy and stiffness in essential hypertensive patients. Hypertension 26: 355-362, 1995.

LAURENT S, GIRERD X, MOURAD J, LACOLLEY P, BECK L, BOUTOUYRIE P: Elastic modulus of the radial artery wall material is not increased in patients with essential hypertension. Arteriosclers Thromb 14: 1223-1231, 1994.

MAILLARD LC: Action of amino acids on sugars. Formation of melanoidins in a methodical way (in French). C R. Seances Acad Sci 154: 66-68, 1912.

MARIN-GARCIA J, GOLDENTHAL MJ: Fatty acid metabolism in cardiac failure: biochemical, genetic and cellular analysis. Cardiovasc Res 54: 516-527, 2002.

MCCANCE DR, DYER DG, DUNN JA, BAILIE KE, THORPE SR, BAYNES JW: Maillard reaction products and their relation to complications in insulin-dependent diabetes mellitus. J Clin Invest 91: 2470-2478, 1993.

MIKŠÍK I, DEYL Z: Post-translational non-enzymatic modification of proteins 2. Separation of selected protein species after glycation and other carbonyl-mediated modifications. J Chromatogr B 699: 311-345, 1997.

MIKŠÍK I, ZICHA J, KUNEŠ J, DEYL Z: Glycation of collagen in hypertriglyceridemic rats. Life Sci 60: 2119-2127, 1997.

MIZUTANI K, IKEDA K, KAWAI Y, YAMORI Y: Biomechanical properties and chemical composition of the aorta in genetic hypertensive rats. J Hypertens 17: 481-487, 1999.

MONNIER VM: Toward a Maillard reaction theory of aging. Prog Clin Biol Res. 304: 1-22, 1989.

MONNIER VM, SELL DR, MIYATA S, NAGARAJ RH, ODETTI P, LAPOLLA A: Advanced Maillard reaction products as markers for tissue damage in diabetes and uraemia: relevance to diabetic nephropathy. Acta Diabetol 29: 130-135, 1992.

NYENGAARD JR, CHANG K, BERHORST S, REISER KM, WILLIAMSON JR, TILTON RG: Discordant effects of guanidines on renal structure and function and on regional vascular dysfunction and collagen changes in diabetic rats Diabetes 46: 94-106, 1997.

REDDY KG: AGE-related cross-linking of collagen is associated with aortic wall matrix stiffness in the pathogenesis of drug-induced diabetes in rats. MicrovascRes 68: 132-142, 2004.

SELL DR, MONNIER VM: Structure elucidation of a senescence cross-link from human extracellular matrix. $J$ Biol Chem 264: 21597-21602, 1989.

SCHNIDER SL, KOHN RR: Effects of age and diabetes mellitus on the solubility and nonenzymatic glucosylation of human skin collagen. J Clin Invest 67: 1630-1635, 1981.

SCHNIDER SL, KOHN RR: Effects of age and diabetes mellitus on the solubility of collagen from human skin, tracheal cartilage and dura mater. Exp Gerontol 17: 185-194, 1982.

SONESSON B, HANSEN F, LANNE T: The mechanical properties of elastic arteries in Ehlers-Danlos syndrome. Eur J Vasc Endovasc Surg 14: 258-264, 1997.

ŠTOLBA P, DOBEŠOVÁ Z, HUŠEK P, OPLTOVÁ H, ZICHA J, VRÁNA A, KUNEŠ J: The hypertriglyceridemic rat as a genetic model of hypertension and diabetes. Life Sci 51: 733-740, 1992.

TRIVELLI LA., RANNEY HM, LAI HT: Hemoglobin components in patients with diabetes mellitus. $N$ Engl J Med 284: 353-357, 1971.

VOGEL HG: Species differences of elastic and collagenous tissue - influence of maturation and age. Mech Ageing Dev57: 15-24, 1991.

VRÁNA A, KAZDOVÁ L: The hereditary hypertriglyceridemic nonobese rat: an experimental model of human hypertriglyceridemia. Transplant Proc 22: 2579, 1990.

WAUTIER JL, GUILLAUSSEAU PJ: Advanced glycation end products, their receptors and diabetic angiopathy. Diabetes Metab 27: 535-542, 2001. 